\title{
Interventional radiology approaches for intra-hepatic cholangiocarcinoma
}

\author{
Cristina Mosconi ${ }^{1}$, Marco Calandri ${ }^{2}$, Milind Javle $^{3}$, Bruno C. Odisio ${ }^{4}$ \\ ${ }^{1}$ Radiology Unit, Department of Specialized, Diagnostic and Experimental Medicine, DIMES, University Hospital of Bologna Sant'Orsola-Malpighi \\ Polyclinic, Alma Mater Studiorum, University of Bologna, Bologna, Italy; ${ }^{2}$ Radiology Unit, Department of Oncology, University of Turin, A.O.U. \\ San Luigi Gonzaga di Orbassano, Turin, Italy; ${ }^{3}$ Department of Gastrointestinal Medical Oncology, ${ }^{4}$ Department of Interventional Radiology, The \\ University of Texas MD Anderson Cancer Center, Houston, TX, USA \\ Contributions: (I) Conception and design: BC Odisio, M Javle; (II) Administrative support: None; (III) Provision of study materials or patients: None; \\ (IV) Collection and assembly of data: None; (V) Data analysis and interpretation: C Mosconi, M Calandri, BC Odisio; (VI) Manuscript writing: All \\ authors; (VII) Final approval of manuscript: All authors. \\ Correspondence to: Bruno C. Odisio, MD. Department of Interventional Radiology, The University of Texas MD Anderson Cancer Center, 1400 \\ Pressler St. FCT 14.5084, Houston, TX 77030, USA. Email: bcodisio@mdanderson.org.
}

\begin{abstract}
The use of loco-regional therapies for the management of patients with biliary tract cancer relies in addressing tumor growth and treat its local complications. This aims to reduce the risk of tumor-related liver failure, the most common cause of death among patients with intra-hepatic cholangiocarcinoma (ICC). Interventional radiology plays a critical role on the management of patients with ICC by providing locoregional oncological, palliative, and bridging procedures for definitive therapy. In regards the oncological procedures offered by interventional radiology, current literature reports its main application for patients with ICC, with limited number of reports on the use of patients with extra-hepatic cholangiocarcinoma. In this review, we aim to provide a literature review on the use of interventional radiology procedures, specifically liver ablation, transarterial chemoembolization (TACE), and transarterial radioembolization (TARE) with $\mathrm{Y}^{90}$ for the loco-regional oncological treatment of patients with ICC. Finally, future directions on the role of such loco-regional oncological treatments offered by interventional radiology are discussed.
\end{abstract}

Keywords: Interventional radiology; cholangiocarcinoma; radioembolization; ablation; chemoembolization

Submitted Aug 09, 2019. Accepted for publication Dec 05, 2019.

doi: $10.21037 /$ cco.2019.12.15

View this article at: http://dx.doi.org/10.21037/cco.2019.12.15

\section{Introduction}

The use of loco-regional therapies for the management of patients with biliary tract cancer has its rational in addressing tumor growth and its local complications. This aims to reduce the risk of tumor-related liver failure, the most common cause of death among patients with intrahepatic cholangiocarcinoma (ICC) $(1,2)$. The use of interventional radiology procedures on the management of patients with biliary tract cancers can be broadly divided in two major goals: loco-regional oncological treatments and non-oncological palliative and/or complementary procedures for definitive therapy. For the former, interventional radiologists apply different loco-regional methods that aims to provide effective local oncological control, therefore preventing tumor progression and its associated complications. Such oncological therapies are mainly applied for patients with ICC, with little evidence on the literature on the use of interventional radiology procedures for the oncological treatment of extrahepatic cholangiocarcinoma; for the latter, interventional radiology procedures are performed to address tumorrelated complications such as bleeding (i.e., transarterial embolization) biliary obstruction (i.e., percutaneous biliary drainage) or as a complementary method to definitive therapy (i.e., pre-operative portal vein embolization). Such 
methods have been successfully applied for the management of both intra- and extra-hepatic cholangiocarcinoma patients.

In this review, we aim to provide a literature review on the use of different interventional radiology procedures for the loco-regional oncological treatment of patients with ICC. Palliative and complementary methods to definitive therapy are not in the scope of the present review and were not included in this review. Finally, future directions on the role of such loco-regional oncological treatments offered by interventional radiology are discussed.

\section{Liver ablation}

The use of image-guided thermal ablation on the treatment of ICC was initially reported two decades ago in a mixed series of primary liver cancer treatments. Specific application of thermal ablation for the ICC was reported for the first time in 2002 by Slakey (3). Although ICC is the second most common liver tumor after hepatocellular carcinoma, due to its relatively low incidence and advanced tumor burden once diagnosed (4), data on the efficacy and safety of thermal ablation in this specific setting are limited if compared to other primary and secondary tumors affecting the liver. Indeed, contrarily to its curative role on patients with hepatocellular carcinoma classified as very early and early stages according to the Barcelona Clinic Liver Cancer (BCLC) Staging and treatment classification (5), thermal ablation is still considered a palliative treatment in the ICC guidelines (6).

The most commonly utilized imaging-guidance modalities to perform liver ablation consists of ultrasound in Europe and Eastern countries and computed tomography (CT) in the United States. During imageguided ablation, thermal energy is delivered to the tumor tissue through needle electrodes (in case of radiofrequency ablation, RFA) or antennas [in case of microwave ablation (MWA)]. In both cases the treatment goal is to reach an intra-tumoral temperature between 60 and $100{ }^{\circ} \mathrm{C}$, to obtain coagulative necrosis. Although RFA has been the most studied ablation modality for ICC, recent evidences support MWA as a valid alternative (7). Indeed, MWA offers many theoretical advantages, including less dependence on electrical conductivity of tissue, a reduced time of ablation, higher intra-tumoral temperature, and larger ablation zone $(8,9)$.

Ablation can be offered for patients with ICC who are not eligible to surgery with small, localized tumors without evidence of extrahepatic spread (3,10-12). Furthermore, due to high recurrence rates of ICC following surgery, ablation has a relevant role in post-resection recurrence $(10,13,14)$. Zhang et al. reported the role of repeated resection $(n=32)$ and thermal ablation $(n=77)$ in patients with ICC presenting with post-resection recurrence. Authors found no differences $(\mathrm{P}=0.99)$ in overall survival (OS) and disease-free survival rates between repeated resection and thermal ablation. Nevertheless, in a subset of patients with recurrence tumors $>3 \mathrm{~cm}$, repeated resection provided improved OS when compared to thermal ablation. Expectedly, major complications were more common in the repeated resection group $(\mathrm{P}<0.001)$. Based on their findings, authors concluded that thermal ablation should be preferred in patients with ICC recurrence measuring $\leq 3 \mathrm{~cm}$ (13).

Lesion size is a relevant factor for achieving complete ablation and reduced rates of local recurrence, also known on the ablation literature as local tumor progression (LTP). It has been suggested a maximum diameter of $3 \mathrm{~cm}$ as a threshold for ablation eligibility for both primary and recurrent ICC (6), which is consistent with the existing liver ablation literature for other tumors types. Furthermore, the $3 \mathrm{~cm}$ cut-off has been identified in the surgical literature to distinguish anatomo-pathological findings, such as a lower incidence of well-differentiated cholangiocarcinoma without microvascular invasion from poorly differentiated tumors with microvascular invasion (15).

Minimal ablation margins is a relevant factor associated with ablation outcomes of patients with HCC and colorectal liver metastasis. Presently, the literature is still lacking of specific and pertinent information regarding the impact of minimal ablation margins on ICC. However, data from surgical series suggests a relevant role for resection margins in order to reduce the local recurrence rates, suggesting a desirable $10 \mathrm{~mm}$ threshold (16). Finally, other factors such as nodes positivity (17), superficially located tumors (18) or presence of extrahepatic disease (15) have been reported to affect outcomes following ablation. Nevertheless, such elements still require more robust confirmation in larger patient cohorts.

A systematic review and meta-analysis of RFA in the treatment of unresectable ICC was performed by Han et al. (11). This included seven observational studies with a total of 84 patients. They found an LTP pooled rates of $21 \%$; the lowest LTP rates was reported by Haidu et al. $(8 \%)$ whereas Carrafiello et al. reported the highest LTP rates of 50\%. Pooled 1-, 3-, and 5-year survival rates were $82,47 \%$ and $24 \%$. On the whole, these findings are 
encouraging, since similar rates were found in metanalysis of thermal ablation of other tumor types, such as colorectal liver metastases. MWA ablation is a more recent technology and few reports are present in the literature. However, in the largest published series (107 patients, 171 treated lesions), Zhang et al. (19) found a median PFS after MWA of 8.9 months. The median OS was 28 months; OS rates after 1,3 and 5 years were $93.5 \%, 39.6 \%$ and $7.9 \%$, respectively (19).

Of the published studies, major complications (i.e., any event that required additional treatment, including an increased level of care, hospital stay beyond observation and permanent damage) were reported at least in 4 studies with one case of death after liver abscess and subsequent sepsis. However, when compared to surgery, complications rates of thermal ablation treatments were far below (13). Minor adverse events, such as post ablation syndrome and abdominal pain, are always reported in all series, but are generally resolved spontaneously or with conservative care.

\section{Transarterial chemoembolization (TACE)}

Conventional TACE (cTACE) is the most utilized intraarterial therapy for patients with ICC. On this procedure, an emulsion of chemotherapeutics and an oil-based contrast agent (Ethiodol or Lipiodol) followed by an embolizing agent is injected into the tumor-supplying branch of the hepatic artery. Doxorubicin, cisplatin and mitomycin-C and, to a lesser extent, gemcitabine is the most commonly used drug combination in the Europe and United States $(20,21)$. Inclusion and exclusion criteria for cTACE are not different from those for patients with hepatocellular carcinoma. In particular, patients should have a good hepatic function (Child-Pugh Class A or B) and appropriate performance status [Eastern Cooperative Oncology Group (ECOG) 0-2]. In cases where tumorrelated biliary occlusion is seen or in patients with prior history of bilioenteric manipulation, there might be a high-risk to develop postprocedural biliary sepsis and liver abscess formation due to chronic biliary colonization with intestinal bacterial flora (22). In such circumstances, aggressive prophylactic antibiotic regimen might be used to prevent infectious complications (23).

Compared with symptomatic management, it has been demonstrated that cTACE is able to improve survival versus best supportive care. Also, Scheuermann et al. demonstrated similar survival between patients with unresectable ICC undergoing cTACE or TACE with drug-eluting beads
(DEB-TACE) (11 months) and patients undergoing surgery with positive resection margins (11 months) or lymph node positive disease (9 months) (24). Published series of patients with unresectable ICC treated with cTACE reported survival from the diagnosis ranging from 12 to 25.2 months and survival from the procedure ranging from 9.1 to 16.3 months (25). However, these studies are characterized by great variability in regard to several variables such as tumor burden, chemotherapeutic agent administered, and number of cTACE sessions. Kiefer et al. (20), in a multicentre study with 62 patients who received cTACE with mitomycin $\mathrm{C}$, doxorubicin, and cisplatin, showed a median time to progression of 8 months, a median OS of 15 months from the first cTACE and 20 months from the diagnosis: patients treated with systemic chemotherapy and cTACE, had a median OS of 28 months (20). Sixty six percent of the 45 patients with at least one follow-up showed disease stability while $10 \%$ showed partial response. Conversely, Kuhlmann et al. demonstrated significantly lower results, reporting an OS of 5.7 months and a progression-free survival (PFS) of only 1.8 months with single-agent cTACE (26). Recently, Ray et al. (27) performed a metaanalysis regarding the efficacy of chemotherapy-based transarterial therapies, demonstrating a cumulative median OS from date of diagnosis of 15.7 months, and from the first TACE treatment of 13.4 months. However, this study had some limitations, in particular the non-uniformity of data about type of chemotherapies and the use and type of the embolic agent; despite the above limitations, in the absence of randomized studies, its results demonstrated the efficacy and safety of TACE in the ICC treatment, being the point of reference in this setting. Generally, cTACE is well tolerated with minimal adverse events. The more frequently minor side effects are related to postembolization syndrome, represented by transient nausea, abdominal pain, fever and increase in liver enzymes (limited to 3-4 days without the evidence of sepsis) (28), which can be usually successfully managed with medical therapy.

Drug-eluting beads transarterial chemoembolization (DEB-TACE) combines the local release of chemotherapeutic agent(s) from beads and arterial embolization with a decrease of blood flow due to the beads, with lower systemic exposure to the chemotherapeutic agent when compared conventional TACE (29). The studies published with inoperable ICC patients treated with DEB-TACE reported survival from the procedure ranged from 8.6 to 30 months (25). However, the same considerations regarding the non-uniformity of the cTACE data may be acknowledged. 


\section{Transarterial radioembolization (TARE)}

The term TARE defines the use of intra-arterially injected radioactive microspheres. The purpose of TARE is to selectively release a high radiation dose specifically to liver tumors and to limit radiation to the normal liver parenchyma to within tolerable levels (30). Following the administration in the hepatic artery, the microspheres are carried by the arterial blood flow toward the most distal arterioles inside hepatic tumors. In addition, the neovascularization of the plexus surrounding the tumor causes an increase density in ICC as compared to the liver parenchyma (31), thus allowing an entrapment of the microspheres in the tumors. For TARE selection, all patients should be evaluated by a multidisciplinary group, analyzing clinical history, physical examination, and of liver function. In addition, recent and high quality radiologic images [CT or magnetic resonance imaging (MRI)] are needed, showing unequivocal and measurable evidence of ICC which cannot be surgically resected or ablated with curative intent (32). The best candidates for TARE are patients with unresectable liver-only or liverdominant tumors (31). The inclusion criteria are: Eastern Cooperation Oncology Group (ECOG) performance status of 0,1 or 2 ; adequate laboratory tests, in particular absolute neutrophil count $>1.53 \times 10^{9} / \mathrm{L}$, a platelet count $>503 \times 10^{9} / \mathrm{L}$, renal function with creatinine level $<2.0 \mathrm{mg} / \mathrm{dL}$, and adequate liver function with bilirubin $<2.0 \mathrm{mg} / \mathrm{dL}$; and 5) the possibility to perform angiographic study of the liver vessels. Exclusion criteria include presence of shunting with gastrointestinal arteries that cannot be embolized; lungs estimated radiation doses greater than $30 \mathrm{~Gy}$, and presence of extra hepatic metastasis representing an imminent lifethreatening outcome.

Ibrahim et al. (33) reported the first results of TARE in 24 patients with histologically proven ICC, showing median OS of 14.9 months from the first treatment. Median survival was significantly longer in patients with ECOG performance status 0 than in those with status 1 and $2(31.8$ vs. 6.1 months and 1 month, respectively, $\mathrm{P}<0.0001)$ and in patients without PVT (31.8 vs. 5.7 months, $\mathrm{P}=0.0003$ ); patients with peripheral ICC had a better survival versus those with infiltrative ICC (31.8 months vs. 5.7 months, respectively, $\mathrm{P}=0.0005)$. A second and more recent study of the same group (34) confirmed these initial results; survival varied according to the multifocality $(5.7 \mathrm{vs} .14 .6$ months, $\mathrm{P}<0.01)$, infiltrative vs. peripheral ICC (6.1 vs. 15.6 months, $\mathrm{P}<0.01)$ or bilobar vs. unilobar disease (10.9 vs. 11.7 months,
$\mathrm{P}=0.37)$.

In a series of 33 patients, Hoffmann et al. (35) reported good results, showing a median OS of 22 months from the initial TARE treatment: survival was significantly improved in patients with ECOG 0, in those with a tumor burden $\leq 25 \%$ and in those with tumor response. Saxena et al. (36) demonstrated similar results, showing a median OS of 9.3 months, significantly correlated to two factors, peripheral tumor type ( $v$ s. infiltrative, $\mathrm{P}=0.004$ ) and an ECOG performance status $(\mathrm{P}<0.001)$. Contrarily, Rafi et al. (37) showed a median OS from the first TARE of $345 \pm 128$ days but not correlated with the ECOG performance status or extra-hepatic metastases. Al-Adra et al. (38) carried out a comprehensive review of the current studies and clinical outcomes of unresectable ICCs treated with TARE, identifying 12 studies with relevant data. Based on a pooled analysis, they found overall weighted median survival was 15.5 months. However, the authors pointed out the heterogeneity of the study populations included in the pooled OS; in fact, all the studies reported survival since the initiation of TARE but, in some cases, the patients had undergone systemic chemotherapy prior to or during the treatment. Therefore, the overall pooled survival may underestimate the effects of TARE if some patients had already undergone previous therapies.

Recently, Edeline et al. (39) and Mosconi et al. (40) showed that TARE combined with chemotherapy seems a promising strategy as first-line treatment for unresectable intrahepatic cholangiocarcinoma; in particular, Mosconi et al. reported a median OS of 17.9 months (95\% CI: 14.3-21.4 months) with better significantly median survival in the treatment-naive patients compared with no naive patients (52 vs. 16 months, $\mathrm{P}=0.009$ ). However, it should be acknowledged a considerable heterogeneity of data reported by the multiple studies in relation to baseline clinical features and tumor type and consequently median survivals ranging from 9 to 22 months; in fact, to date, indications of TARE included patients with peripheral and infiltrative type, different tumor burden, as well as patients who received previous treatment or not or concomitant chemotherapy. Cucchetti et al. (41) recently performed a meta-regression study, obtaining two very relevant results: first, naïve patients had a 2 -year survival of $50.4 \%$ vs. $23.6 \%$ of patients treated with TARE after failure/recurrence of a previous treatment, with statistically significant difference; second, they reported a median pooled survival of 19.3 months in patients with peripheral ICC, considerably higher than the infiltrative type estimated to be 8.2 months. 
However, these results are not confirmed by recently paper of Gangi et al. which includes 85 patients resulting in the most numerous cases published (42); in this paper, median OS from radioembolization was 12.0 months and it was not significantly longer in treatment-naïve patients or with mass-forming type.

The complications following TARE are not frequent and can be further decreased by strict pretreatment assessment and carefully selecting patients with preserved liver function; post-radioembolization syndrome (PRS), hepatic dysfunction, biliary sequelae, portal hypertension, radiation pneumonitis, gastrointestinal (GI) ulceration, vascular injury and lymphopenia are, even if rare, the most frequently adverse events (43). TARE is important therapeutic option, with good results about OS and low incidence of complications, for patients with unresectable ICC; future multicenter randomized trials are necessary to understand the role and timing of TARE and other palliative therapies in the management of this cancer.

\section{Future directions and conclusions}

Despite of the existing evidence that the use of locoregional therapies for the management of ICC might provide effective local tumor control with limited sideeffects, its utilization on clinical practice is still limited to a small number of patients. Amini et al. (44), performed an evaluation of 5,388 patients with ICC using the Surveillance Epidemiology and End Results. In their analysis, authors demonstrated that only $23 \%$ of the patients with ICC were submitted to local therapies, with surgery being the most commonly utilized. Unfortunately, only one-third of the patients with ICC are eligible for surgical resection at the time of the diagnosis (45). In this context, the use of loco-regional oncological therapies by interventional radiology might potentially beneficiate a significant amount of patients who are ineligible for curative resection and further prospective studies should be encouraged.

The use of liver ablation, although confined to patients with ICC who present with limited disease who are otherwise not surgical candidates, has increased nearly six fold in the last three decades (44). The wider availability of ablation technologies along with its technical refinements will likely provide a further increase on the use of ablation on patients with ICC. More importantly, validation of technical and clinical predictive factors, in particular better understanding of tumor molecular biology, will hopefully improve patient selection for ablation and its outcomes. Similarly, recent improvements on the understanding of tumor dose response following TARE (46) will allow improving patient selection and refining treatment strategies to increase tumor response rates, while preserving non-tumorous normal liver parenchyma from the deleterious effects of radiation. Finally, the aggressive and multicentric nature of ICC requires the need of using distinct therapies in a sequential and combined approach (Figure 1), highlighting the need of multidisciplinary management of such patients for maximum oncological control, while preserving liver function and quality of life. 

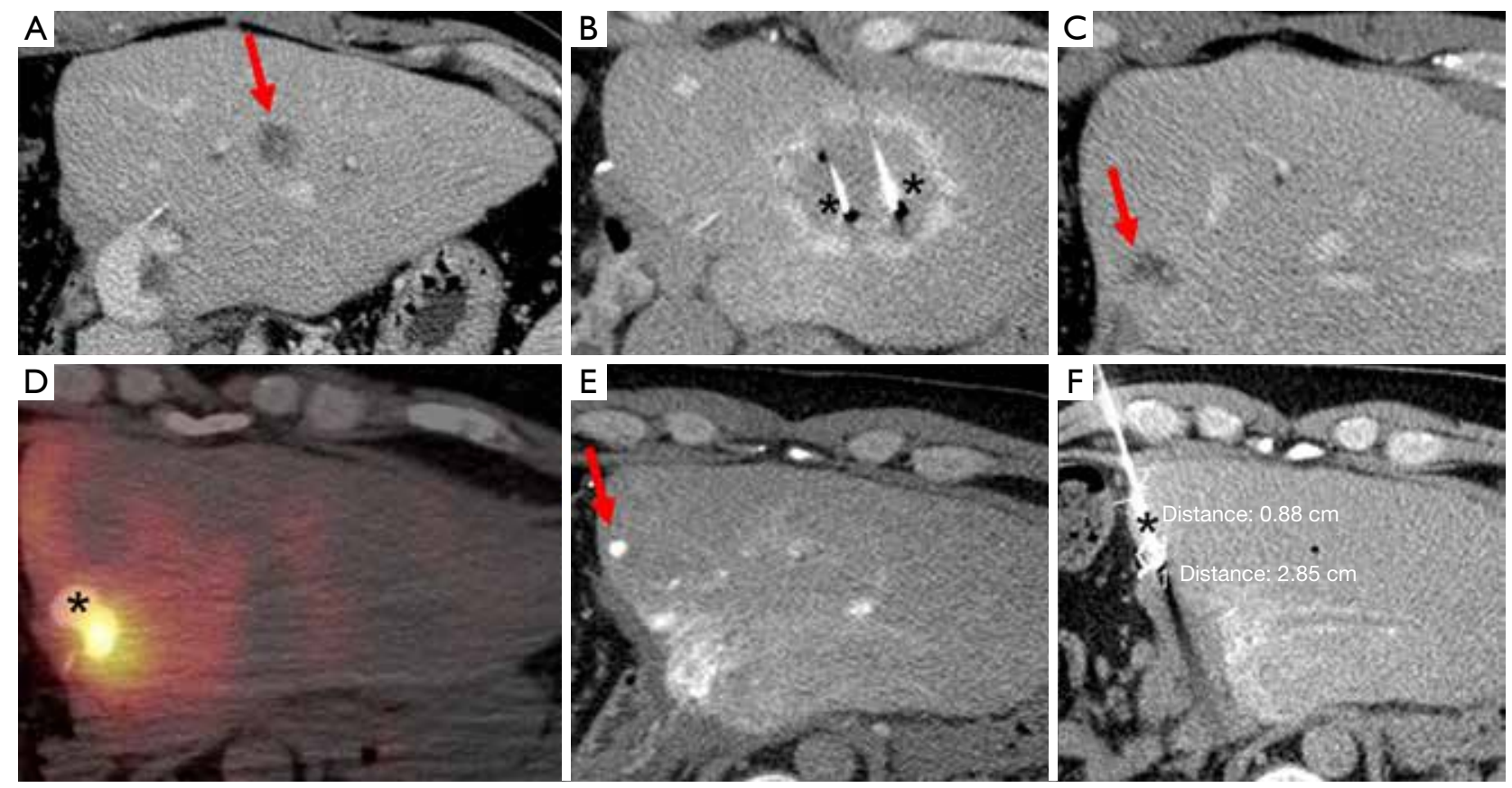

Figure 1 Example on the use of sequential loco-regional therapies on a 38-year-old male patient presenting with ICC on the right hepatic lobe (not shown) status systemic chemotherapy with Gemcitabine and Cisplatin followed by right hepatectomy; (A) 22 months following hepatectomy patient developed tumor recurrence (arrow) within the left hepatic lobe; (B) successful ablation of the tumor was performed under CT-guidance ( ${ }^{*}$, ablation probes); (C) another tumor (arrow) was also noted close to the main left hepatic bile duct, precluding the use of ablation; (D) decision was made to treat tumor with $\mathrm{Y}^{90}$ radioembolization (*, bremsstrahlung PET CT showing contrast and radiation deposition within the target lesion); (E) 4 months later patient presented with new tumor (arrow) at the surgical margin; (F) ablation was carried out to that lesion (*, ablation probes). No tumor progression was noted following such therapies for a 7-month period. Patient was enrolled on the liver transplant waitlist and submitted to cadaveric liver transplant. Liver explant showed absence of viable tumor in the liver (not shown).

\section{Acknowledgments}

None.

\section{Footnote}

Conflicts of Interest: BC Odisio: Research Grant, Siemens Healthineers. The other authors have no conflicts of interest to declare.

Ethical Statement: The authors are accountable for all aspects of the work in ensuring that questions related to the accuracy or integrity of any part of the work are appropriately investigated and resolved.

\section{References}

1. Tao R, Krishnan S, Bhosale PR, et al. Ablative
Radiotherapy Doses Lead to a Substantial Prolongation of Survival in Patients With Inoperable Intrahepatic Cholangiocarcinoma: A Retrospective Dose Response Analysis. J Clin Oncol 2016;34:219-26.

2. Patel T. Cholangiocarcinoma--controversies and challenges. Nat Rev Gastroenterol Hepatol 2011;8:189-200.

3. Slakey DP. Radiofrequency ablation of recurrent cholangiocarcinoma. Am Surg 2002;68:395-7.

4. Kudo M, Izumi N, Ichida T, et al. Report of the 19th follow-up survey of primary liver cancer in Japan. Hepatol Res 2016;46:372-90.

5. Forner A, Reig M, Bruix J. Hepatocellular carcinoma. Lancet 2018;391:1301-14.

6. Bridgewater J, Galle PR, Khan SA, et al. Guidelines for the diagnosis and management of intrahepatic cholangiocarcinoma. J Hepatol 2014;60:1268-89.

7. Yu MA, Liang P, Yu XL, et al. Sonography-guided percutaneous microwave ablation of intrahepatic primary 
cholangiocarcinoma. Eur J Radiol 2011;80:548-52.

8. Wright AS, Sampson LA, Warner TF, et al.

Radiofrequency versus microwave ablation in a hepatic porcine model. Radiology 2005;236:132-9.

9. Yu J, Liang P, Yu X, et al. A comparison of microwave ablation and bipolar radiofrequency ablation both with an internally cooled probe: results in ex vivo and in vivo porcine livers. Eur J Radiol 2011;79:124-30.

10. Kim JH, Won HJ, Shin YM, et al. Radiofrequency ablation for recurrent intrahepatic cholangiocarcinoma after curative resection. Eur J Radiol 2011;80:e221-5.

11. Han K, Ko HK, Kim KW, et al. Radiofrequency ablation in the treatment of unresectable intrahepatic cholangiocarcinoma: systematic review and meta-analysis. J Vasc Interv Radiol 2015;26:943-8.

12. Butros SR, Shenoy-Bhangle A, Mueller PR, et al. Radiofrequency ablation of intrahepatic cholangiocarcinoma: feasability, local tumor control, and long-term outcome. Clin Imaging 2014;38:490-4.

13. Zhang SJ, Hu P, Wang N, et al. Thermal ablation versus repeated hepatic resection for recurrent intrahepatic cholangiocarcinoma. Ann Surg Oncol 2013;20:3596-602.

14. Kamphues C, Seehofer D, Eisele RM, et al. Recurrent intrahepatic cholangiocarcinoma: single-center experience using repeated hepatectomy and radiofrequency ablation. J Hepatobiliary Pancreat Sci 2010;17:509-15.

15. Spolverato G, Kim Y, Ejaz A, et al. Conditional Probability of Long-term Survival After Liver Resection for Intrahepatic Cholangiocarcinoma: A Multi-institutional Analysis of 535 Patients. JAMA Surg 2015;150:538-45.

16. Li MX, Bi XY, Li ZY, et al. Impaction of surgical margin status on the survival outcome after surgical resection of intrahepatic cholangiocarcinoma: a systematic review and meta-analysis. J Surg Res 2016;203:163-73.

17. Xu HX, Wang Y, Lu MD, et al. Percutaneous ultrasound-guided thermal ablation for intrahepatic cholangiocarcinoma. Br J Radiol 2012;85:1078-84.

18. Takahashi EA, Kinsman KA, Schmit GD, et al. Thermal ablation of intrahepatic cholangiocarcinoma: Safety, efficacy, and factors affecting local tumor progression. Abdom Radiol (NY) 2018;43:3487-92.

19. Zhang K, Yu J, Yu X, et al. Clinical and survival outcomes of percutaneous microwave ablation for intrahepatic cholangiocarcinoma. Int J Hyperthermia 2018;34:292-7.

20. Kiefer MV, Albert M, McNally M, et al. Chemoembolization of intrahepatic cholangiocarcinoma with cisplatinum, doxorubicin, mitomycin C, ethiodol, and polyvinyl alcohol: a 2-center study. Cancer
2011;117:1498-505.

21. Shen WF, Zhong W, Liu Q, et al. Adjuvant transcatheter arterial chemoembolization for intrahepatic cholangiocarcinoma after curative surgery: retrospective control study. World J Surg 2011;35:2083-91.

22. Padia SA. Intrahepatic Cholangiocarcinoma. Tech Vasc Interv Radiol 2015;18:227-35.

23. Odisio BC, Richter M, Aloia TA, et al. Use of Prophylactic Antibiotics to Prevent Abscess Formation Following Hepatic Ablation in Patients with Prior Enterobiliary Manipulation. J Gastrointest Surg 2016;20:1428-34.

24. Scheuermann U, Kaths JM, Heise M, et al. Comparison of resection and transarterial chemoembolisation in the treatment of advanced intrahepatic cholangiocarcinoma-a single-center experience. Eur J Surg Oncol 2013;39:593-600.

25. Ierardi AM, Angileri SA, Patella F, et al. The role of interventional radiology in the treatment of intrahepatic cholangiocarcinoma. Med Oncol 2017;34:11.

26. Kuhlmann JB, Euringer W, Spangenberg HC, et al. Treatment of unresectable cholangiocarcinoma: conventional transarterial chemoembolization compared with drug eluting bead-transarterial chemoembolization and systemic chemotherapy. Eur J Gastroenterol Hepatol 2012;24:437-43.

27. Ray CE, Jr., Edwards A, Smith MT, et al. Metaanalysis of survival, complications, and imaging response following chemotherapy-based transarterial therapy in patients with unresectable intrahepatic cholangiocarcinoma. J Vasc Interv Radiol 2013;24:1218-26.

28. Cohen MJ, Bloom AI, Barak O, et al. Trans-arterial chemo-embolization is safe and effective for very elderly patients with hepatocellular carcinoma. World J Gastroenterol 2013;19:2521-8.

29. Aliberti C, Benea G, Tilli M, et al. Chemoembolization (TACE) of unresectable intrahepatic cholangiocarcinoma with slow-release doxorubicin-eluting beads: preliminary results. Cardiovasc Intervent Radiol 2008;31:883-8.

30. Kennedy A, Coldwell D, Sangro B, et al. Radioembolization for the treatment of liver tumors general principles. Am J Clin Oncol 2012;35:91-9.

31. Kennedy A, Nag S, Salem R, et al. Recommendations for radioembolization of hepatic malignancies using yttrium-90 microsphere brachytherapy: a consensus panel report from the radioembolization brachytherapy oncology consortium. Int J Radiat Oncol Biol Phys 2007;68:13-23.

32. Coldwell D, Sangro B, Wasan H, et al. General selection criteria of patients for radioembolization of liver tumors: 
an international working group report. Am J Clin Oncol 2011;34:337-41.

33. Ibrahim SM, Mulcahy MF, Lewandowski RJ, et al. Treatment of unresectable cholangiocarcinoma using yttrium-90 microspheres: results from a pilot study. Cancer 2008;113:2119-28.

34. Mouli S, Memon K, Baker T, et al. Yttrium-90 radioembolization for intrahepatic cholangiocarcinoma: safety, response, and survival analysis. J Vasc Interv Radiol 2013;24:1227-34.

35. Hoffmann RT, Paprottka PM, Schon A, et al. Transarterial hepatic yttrium- 90 radioembolization in patients with unresectable intrahepatic cholangiocarcinoma: factors associated with prolonged survival. Cardiovasc Intervent Radiol 2012;35:105-16.

36. Saxena A, Bester L, Chua TC, et al. Yttrium-90 radiotherapy for unresectable intrahepatic cholangiocarcinoma: a preliminary assessment of this novel treatment option. Ann Surg Oncol 2010;17:484-91.

37. Rafi S, Piduru SM, El-Rayes B, et al. Yttrium- 90 radioembolization for unresectable standardchemorefractory intrahepatic cholangiocarcinoma: survival, efficacy, and safety study. Cardiovasc Intervent Radiol 2013;36:440-8.

38. Al-Adra DP, Gill RS, Axford SJ, et al. Treatment of unresectable intrahepatic cholangiocarcinoma with yttrium-90 radioembolization: a systematic review and pooled analysis. Eur J Surg Oncol 2015;41:120-7.

39. Edeline J, Du FL, Rayar M, et al. Glass Microspheres $90 \mathrm{Y}$ Selective Internal Radiation Therapy and Chemotherapy as First-Line Treatment of Intrahepatic Cholangiocarcinoma.
Clin Nucl Med 2015;40:851-5.

40. Mosconi C, Gramenzi A, Ascanio S, et al. Yttrium-90 radioembolization for unresectable/recurrent intrahepatic cholangiocarcinoma: a survival, efficacy and safety study. Br J Cancer 2016;115:297-302.

41. Cucchetti A, Cappelli A, Mosconi C, et al. Improving patient selection for selective internal radiation therapy of intra-hepatic cholangiocarcinoma: A meta-regression study. Liver Int 2017;37:1056-64.

42. Gangi A, Shah J, Hatfield N, et al. Intrahepatic Cholangiocarcinoma Treated with Transarterial Yttrium-90 Glass Microsphere Radioembolization: Results of a Single Institution Retrospective Study. J Vasc Interv Radiol 2018;29:1101-8.

43. Riaz A, Lewandowski RJ, Kulik LM, et al. Complications following radioembolization with yttrium-90 microspheres: a comprehensive literature review. J Vasc Interv Radiol 2009;20:1121-30; quiz 1131.

44. Amini N, Ejaz A, Spolverato G, et al. Temporal trends in liver-directed therapy of patients with intrahepatic cholangiocarcinoma in the United States: a populationbased analysis. J Surg Oncol 2014;110:163-70.

45. Dodson RM, Weiss MJ, Cosgrove D, et al. Intrahepatic cholangiocarcinoma: management options and emerging therapies. J Am Coll Surg 2013;217:736-50.e4.

46. Kappadath SC, Mikell J, Balagopal A, et al. Hepatocellular Carcinoma Tumor Dose Response After (90) Y-radioembolization With Glass Microspheres Using (90) Y-SPECT/CT-Based Voxel Dosimetry. Int J Radiat Oncol Biol Phys 2018;102:451-61.
Cite this article as: Mosconi C, Calandri M, Javle M, Odisio BC. Interventional radiology approaches for intra-hepatic cholangiocarcinoma. Chin Clin Oncol 2020;9(1):8. doi: 10.21037/cco.2019.12.15 